\title{
Aqueous Stable Silver Nanoparticles Derived from Nimble Leaves of Psidium Guajava
}

\author{
Keyur D Bhatt*, Yesha R Desai, Nirali K Sharma, Palak C Sukhadiya and Mam- \\ ta R Zalailya
}

Department of Chemistry Ganpat University, India

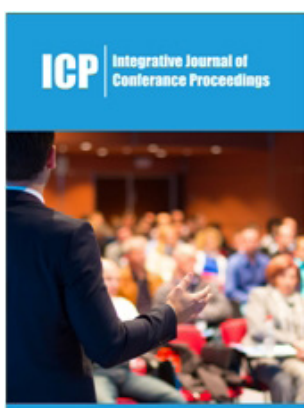

*Corresponding author: Keyur D Bhatt, Department of Chemistry Ganpat University, India

Submission: 侮 May 14, 2019

Published: 眥July 08, 2019

Volume 1 - Issue 4

How to cite this article: Keyur D B, Yesha R D, Nirali K S, Palak C Sukhadiya, Mamta R Z. Aqueous Stable Silver Nanoparticles Derived from Nimble Leaves of Psidium Guajava. Int J Conf Proc.1(4). ICP.000519.2019.

Copyright@ Keyur D Bhatt, This article is distributed under the terms of the Creative Commons Attribution 4.0 International License, which permits unrestricted use and redistribution provided that the original author and source are credited.

\begin{abstract}
Here, we report the synthesis of silver nanoparticles (AgNPs) by utilizing extract of Psidium guajava. In this study leaf was with extricated alcohol, further this extract is utilized for the preparation of nanoparticles followed by treatment with silver nitrate solution. Color change from colorless to dark-yellow and SPR band (UV-Vis) $420 \mathrm{~nm}$ which indicates the synthesis of plant reduced silver nanoparticles. The presences of proteins as capping agent, which increase the stability of AgNPs in the colloids, are characterized by Fourier transform infrared spectroscopy (FTIR). Transmission electron microscopy (TEM) demonstrated the presence at spherical AgNPs in the range of $15-30 \mathrm{~nm}$. The bactericidal activity of standard antibiotics was essentially expanded in the presence of AgNPs against pathogenic bacteria, $S$ Aureus, Staphylococcus, E Coli, P Putida and fungus A Niger.
\end{abstract}

Keywords: Psidium guajava leaves; Silver nanoparticles; FTIR; TEM; Antimicrobial activity

\section{Introduction}

A nanoparticle is a substance with a size of $1-100 \mathrm{~nm}$; containing tens to thousands of particles. The interfacial level generally comprises inorganic and organic particles [1]. The prefix word nano commonly used for particles that have size up to several nanometers [2]. The national nanotechnology initiative defined the nanoparticle as structures have size ranging from 1-100nm in at smallest amount one dimension [3]. Nanoparticles can be easily synthesized utilizing differentstrategies by different methodologies accessible for the synthesis of nanoparticles includes chemical, physical biological metal to synthesize nanoparticles. Nanoparticles are various types. Organic nanoparticles which involve carbon nanoparticles and inorganic nanoparticles involve magnetic nanoparticles, noble nanoparticles like gold and silver and semi-conductor nanoparticles like titanium oxide and zinc oxide. Green synthesis furnish progress over chemical as well as physical techniques it is prize effective, environment friendly, easily scaled for bigger scale synthesis and this strategy no compelling reason utilize high pressure, energy, temperature and harmful materials [4]. Nanoparticles assume a vital part in drug delivery, sensing, gene delivery, artificial implants, diagnostics imaging, and tissue engineering [5]. However, most of the chemical techniques utilize for the synthesis of nanoparticles includes the utilization of harmful, dangerous chemical processes is not ecofriendly [6]. Silver metal nanoparticle has a high particular surface area and a high fraction of surface atoms of the select physicochemical characteristics of nanoparticle, including catalytic activity, optical properties, antibacterial properties, and magnetic properties [7]. The synthesis of silver nanoparticles by utilizing different plant extract for example, Helianthus annuus (sunflower), Basella alba, Oryza sativa (Rice), Pine (Pinus desiflora) ,Mangolia kobus (Mangnolia) and Platanus orientalis (Platanus) leaves, Banana peel, Ocimum sanctum(Tulsi) stems, roots, leaf, Murraya koenigi (Curry) leaf, Mangostana (Mangosteem) leaf, Ocimum tenuiflorum, Azadirachta indica (Neem) [8]. The different plant material and their extract can be utilizing the synthesis of nanoparticles in another biological synthesis process. Plant extract material utilized bacteria, fungi and enzymes for synthesis of silver nanoparticles [9]. Advantage of eco-friendly and compatibility for pharmaceutical and biomedical applications [10]. The biological synthesis of silver nanoparticles utilizing microorganisms as well as bacteria, fungi and plants; on account of their antioxidant or reducing character commonly responsible for the reduction of alloy compound in their separate nanoparticles 
[11]. It is one of the top platforms for synthesis of nanoparticles as it is free from harmful chemical as well as providing natural capping agents for the stabilization of silver nanoparticles [12]. The distinctive parts of plant, for example, stem, root, fruit, seed, callus, peel, leaves and bloom are utilized for synthesis of metallic nanoparticles in different shapes and size by biological approaches. To characterization nanoparticles different procedure like UV-Visible spectrophotometer, Dynamic light scattering (DLS), Scanning electron microscopy (SEM), Transition electron microscopy (TEM), Zeta potential measurement, Fourier transform infrared spectroscopy (FTIR), X-ray diffraction (XRD), Energy dispersive spectroscopy (EDS), Auger electron spectroscopy (AES), Low energy ion scattering (LEIS), Scanning tunneling microscopy (STM) and Atomic force microscopy (AFM). All these procedures are greatly helpful to know the complete description details of synthesized nanoparticles [13]. The potential utilization of nano engineered layers to intensity reactant process could empower higher-, productivity, little-scale fuel cells. The development in portable electronic equipment (cell phones, navigation devices, laptop computers, remote sensors), there is great demand for lightweight, high-energy density batteries [14].

Psidium guajava is generally known as Guava. Its family is Myrtaceae. This fruit are commonly cultivated in tropical and subtropical region. Guava tree is small tree and its native to Mexico, Central America, northern South America and Hawaii. In India guava tree are cultivated in utter Pradesh, Punjab, Bihar, Tamil Nadu, Karnataka, Orissa, Haryana and Maharashtra. Tropical and subtropical both climates are suitable for Guava [15]. Psidium guajava has been utilized as medicinal plant all over the world for a number of elements. The use of guava parts is fresh or dried leaves, bark and fruit. These plants are mainly used in therapeutically [16]. Guava tree can be developed in all type of soil but it sensitive to water logging. Develop trees of most species are cold-hardy and can survive temperature somewhat colder than $\left(-3^{\circ} \mathrm{C}\right)$ for brief timeframes, however younger plants will probably freeze to the ground. The $\mathrm{pH}$ rang of soil must be 5.0 to 7.0 is harmonious for guava. Leaves of guava are oval to oblong-elliptic, evergreen leathery. It has short petioles. At the point when leaves are crushed it gave aromatic smell. Leaves are 10 to 15 centimeters lengthy and lengths are 5 to 7 centimeter wide [17]. The plant has likewise been utilized for the controlling of life-changing condition for example, diabetes, malaria, ulcer, hypertension, and obesity. In leaves, the chemical compounds detected are tannins, flavanoids (quercetin, luteolin), essential oils ( $\boldsymbol{\alpha}$-pinene, 1, 8-cineol), tri-terpenoids oils (ursolic acid, oleanic acid) and $\boldsymbol{\beta}$-sitosterol, polyphenols, flavonoids and tannins are the utilization different of diseases. Fresh leaves are utilized as natural pain reliever. It is antioxidants, antibacterial, anti-inflammatory agents [18].

\section{Experimental}

\section{Chemical and reagents}

All starting materials, Reagents, metal nitrate salts, and other chemicals were obtained from Sigma-Aldrich. Silica gel and fluorescence active TLC plates (F-2009) were purchased from the
Merck. All aqueous solutions were prepared with quartz distilled deionized water, which was further purified by a Millipore Milli-Q water purification system. All the solvents employed for synthesis were commercially available and used as received without further purification.

\section{Instrument}

The melting points (uncorrected) were obtained from a VEEGO Model; VMP-DS, (Mumbai, India). Samples for infrared spectra were prepared as $\mathrm{KBr}$ pallets; spectra were recorded on tensor Bruker 27 (Ettlingen, Germany) and expressed in $\mathrm{cm} 1$. Elemental analysis (C, $\mathrm{H}$ and $\mathrm{N}$ ) was performed on vario MICRO-Variant elemental analyzer (Mt. Laurel, USA). Electrospray ionization (ESI) mass spectra (MS) were determined using Micro mass Quarter2 (Utah, USA). UV-Vis spectra were recorded on a JASCO spectrophotometer (Easton, US).

\section{Collection and Identification}

The plant material was collected in the month of December from Mehsana District. It was identified by department of pharmacognosy, SKPCPER, GNU and herbarium specimen was stored. Dirt particles were removed, and it was subjected for shade drying for 15-20 days. After drying, leaves were separated. After leaves were collected in a tray and it was inspected visibly for solid impurities. After shifting in a sieve, grinding was done, and leaves were converted to fine dry powder. The powder was stored in an airtight container for further use.

\section{Method for extraction}

There is various method of extraction for crude drug like infusion, decoction, digestion, percolation, maceration and hot continuation percolation (Soxhlet). Among all these methods, hot continuous percolation i.e. Soxhlet method is most reliable and it gives maximum yield of the extract.

\section{Procedure}

Take 30gm of Psidium guajava leaves powdered was packed in a thimble which was placed into extractor. In first stage, $500 \mathrm{ml}$ hexane was added into a round bottom flask. By this step, fatty materials and oils were removed. The continuous cycles were carried out until solution become colorless for 4 hours. After that, it was further extracted with $500 \mathrm{ml}$ methanol until the clear white solutions for 3 hours from the siphon tube was obtained. After the Soxhlet extraction, a thick dark solvent with extracted material is accumulated in the round bottom flask. Extra solvent was removed by suction pump. Extract was collected and dried in a porcelain dish and placed into desiccators. This extract was further used for experimental purpose Figure 1 \& 2 .

\section{Synthesis of aqueous stable silver nanoparticles (AgNPs) derived from Psidium guajava}

Heat $50 \mathrm{ml}$ of plant extract [Psidium guajava] solution in $50 \mathrm{ml}$ water at $50{ }^{\circ} \mathrm{C}$ and then add $50 \mathrm{ml} \mathrm{AgNO}_{3}\left(1 \times 10^{-3} \mathrm{M}\right)$. Keep the reaction mixture under vigorous stirring for 1 hour, after which the colorless solution change to dark yellow. The dark-yellow 
color formation and UV-Vis spectroscopy Confirms the successful formation of silver nanoparticles. Therefore, a simple one-pot chemical reduction process using Plant extract [Psidium guajava] as stabilizing, reducing and capping agent in environmentally benign solvent.

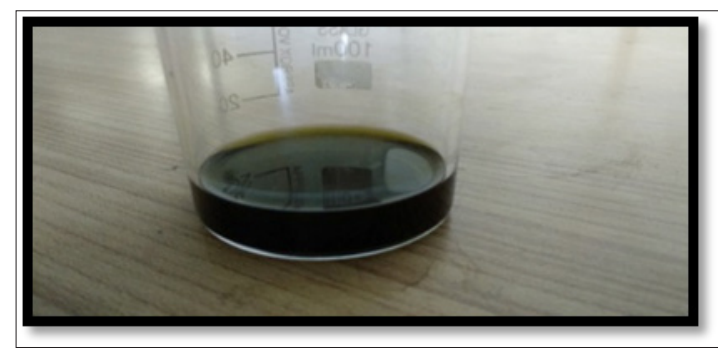

Figure 1: Aqueous leaves extract of Psidium guajava.

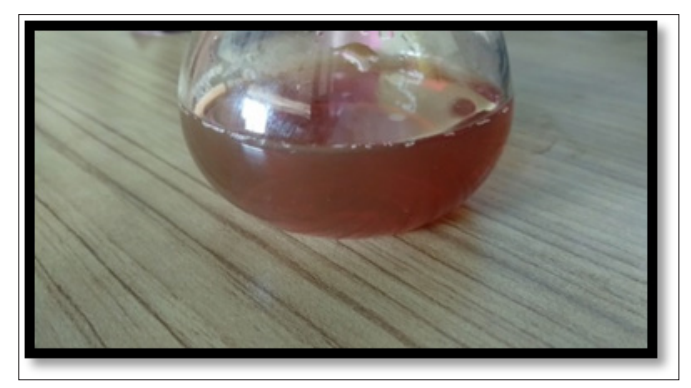

Figure 2: Characterization of plant extract.

\section{Results \& Discussion}

In this work we address the use of plant extract [Psidium guajava] as a reducing and stabilizing agent for formation of AgNPs in an aqueous medium. Reduction of $\mathrm{AgNO}_{3}$ by plant extract [Psidium guajava] occurs via oxidation of the amino group present in chemical constituents of plant i.e., transfer of electrons from the amine of plant extract [Psidium guajava] to the Ag+ ion. The resulting metallic silver nucleates to form silver nanoparticles. The plant extract [Psidium guajava] caped the silver nanoparticles after the reduction process and stabilized it electrostatically.

$$
\mathrm{AgNO}_{3}+\mathrm{NR}_{3} \rightarrow \mathrm{Ag} 0+\mathrm{NR}_{3}
$$

As it is known, for the reduction reaction to proceed, it is essential that the reduction potential of $\mathrm{AgNO}_{3}$ should be lower than the oxidation potential of the amine. When an amine reducing agent has an oxidation potential lower than the reduction potential of $\mathrm{AgNO}_{3}$. Representative TEM image of plant extract [Psidium guajava] the reduced silver nanoparticles as shown in Figure 3 revealed that the particles are roughly spherical in shape reasonably uniform in size and well dispersed with a narrow size distribution with an average particles size of $5 \mathrm{~nm}$. Further these plants extract [Psidium guajava] silver nanoparticles were screened against bacteria, S.Aureus, Staphylococcus, E Coli, P.Putida and Fungus A.Niger. Plant extract [Psidium guajava] silver nanoparticles shows good potent activity against S.Aureus, Staphylococcus, E-Coil, P.Putida. Bacteria and A. Niger fungus.

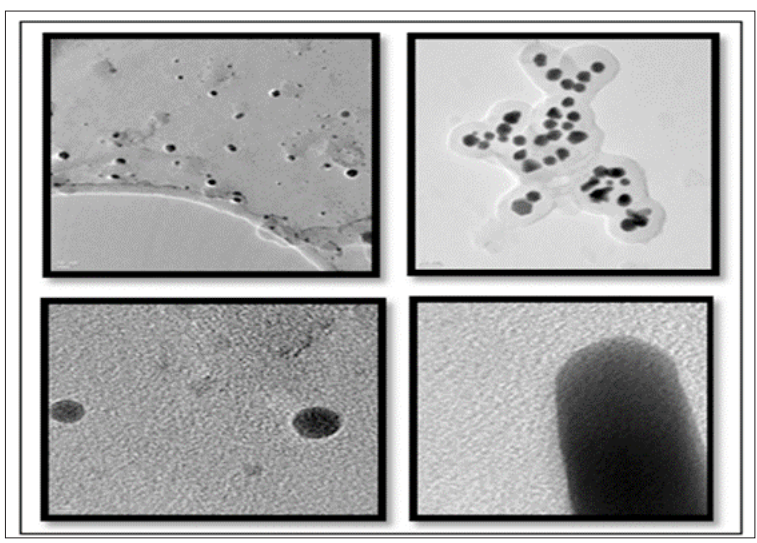

Figure 3: TEM Image of Ag Nanoparticles synthesized from $\mathrm{AgNO}_{3}$ using Psidium Guajava Leaves Extract (Size from 10-35nm).

\section{Conclusion}

Here we report the synthesis of aqueous stable silver nanoparticles by using plant extract [Psidium guajava] as both reducing stabilizing and capping agents plant extract [Psidium guajava] reduced silver nanoparticles were characterized by UV/ Vis, FTIR and transmission electron microscopy (TEM). The records confirmed high stability of Plant extract [Psidium guajava]-AgNPs in aqueous solution over a long period of time and even at varied pH. Additionally, Plant extract [Psidium guajava]-AgNPs have been investigated for its application in antimicrobial activity against gram positive, gram negative and fungus.

\section{References}

1. Geiser M, Rothen R, Kapp B, Schürch N, Kreyling S, et al. (2005) Ultrafine particles cross cellular membranes by nonphagocytic mechanisms in lungs and in cultured cells. Environmental Health Perspectives 113(11): 1555-1560.

2. Nikalje AP (2015) Nanotechnology and its applications in medicine. Med chem 5(2): 81-89.

3. Jain KK, Jain KK (2008) The handbook of nanomedicine. Springer: Vol 404.

4. Sekhon BS (2010) Food nanotechnology-an overview. Nanotechnol Sci Appl 3: 1-15.

5. Yadav SG, Patil SH, Patel P, Nair V, Khan S, et al. (2018) Green synthesis of silver nanoparticles from plant sources and evaluation of their antimicrobial activity 9(1): 1-9.

6. Prabhu S, Poulose EK (2012) Silver nanoparticles: mechanism of antimicrobial action, synthesis, medical applications, and toxicity effects. International Nano Letters 2(1): 32.

7. Muthukumar H, Matheswaran M (2015) Amaranthus spinosus leaf extract mediated $\mathrm{FeO}$ nanoparticles: physicochemical traits, photocatalytic and antioxidant activity. ACS Sustainable Chemistry \& Engineering 3(12): 3149-3156.

8. Awwad AM, Salem NM, Abdeen AO (2013) Green synthesis of silver nanoparticles using carob leaf extract and its antibacterial activity. International Journal Of Industrial Chemistry 4(1): 29.

9. Shah M, Fawcett D, Sharma S, Tripathy SK, Poinern GE (2015) Green synthesis of metallic nanoparticles via biological entities. Materials 8(11): 7278-7308. 
10. Mirzaei H, Darroudi M (2017) Zinc oxide nanoparticles: Biological synthesis and biomedical applications. Ceramics International 43(1) 907-914.

11. Chauhan A, Zubair S, Tufail S, Sherwani A, Sajid M, et al. (2011) Fungusmediated biological synthesis of gold nanoparticles: potential in detection of liver cancer. International Journal of Nanomedicine 6: 23052319.

12. Ahmed S, Ikram S (2015) Silver nanoparticles: one pot green synthesis using terminalia arjuna extract for biological application. J Nanomed Nanotechnol 6(4): 309.

13. Anandalakshmi K, Venugobal J, Ramasamy V (2016) Characterization of silver nanoparticles by green synthesis method using pedalium murex leaf extract and their antibacterial activity. Applied Nanoscience 6(3): 399-408.
14. Nathan A, Ahnood A, Cole MT, Lee S, Suzuki Y (2012) Flexible electronics: the next ubiquitous platform. Proceedings of the IEEE 100: 1486-1517.

15. Chavan U, Processing of Guava into Various Products.

16. Rahim N, Gomes DJ, Watanabe H, Rahman SR, Chomvarin C, et al. (2010) Antibacterial activity of Psidium guajava leaf and bark against multidrug-resistant Vibrio holerae: implication for cholera control. Japanese Journal Of Infectious Diseases 63(4): 271-274.

17. Menzel C (1985) Guava: An exotic fruit with potential in queensland. Queensland Agricultural Journal 111(2): 93-98.

18. Braga TV, Das DR, Ramos CS, Evangelista FC, Silva T, et al. (2014) Antioxidant, antibacterial and antitumor activity of ethanolic extract of the Psidium guajava leaves. American Journal of Plant Sciences 5(23): 3492 .

For possible submissions Click below: 